\title{
Volumetrically Scanning the Structure of Stray-fields Above Grain-Oriented Electrical-Steel Using a Variably Angled TMR Sensor
}

\author{
Robert Gibbs, Turgut Meydan, Paul Williams \\ Wolfson Centre for Magnetics \\ School of Engineering, Cardiff University \\ Cardiff, United Kingdom
}

\begin{abstract}
-a new versatile scanning hardware based on a Micromagnetics $^{\circledR}$ STJ-020 MgO-based tunnelling magnetoresistance (TMR) sensor has been developed to volumetrically scan the thin boundary layer above a given sample. An $(x, y)$ planar scan of the surface of a $7.5 \mathrm{~mm} \times 7.5 \mathrm{~mm}$ sample of grainoriented $(3 \% \mathrm{Si})$ electrical steel is presented. Stray fields normal to the surface between -116 and $272 \mathrm{~A} / \mathrm{m}$ are measured. At 10 $\mu \mathrm{m} /$ pixel domain and micro-domain structures are seen. At 5 $\mu \mathrm{m} /$ pixel the micro-domain structures resolve into clear Lancet domains. The domain images presented have greater qualitative similarity with Kerr effect observations than with Bitter technique results. An $(x, z)$ vertical scan along a $2.35 \mathrm{~mm}$ transect reveals the perpendicular extent of the stray fields, with the normal components shown to emanate from the domain bodies and extend approximately $40-100 \mu \mathrm{m}$ from the sample surface. With the aim of investigating how the stray fields close back onto the surface, the $(x, z)$ transect is repeated with the sensor at 5,10 , 15 and 20 degrees from the vertical. For the first time, the stray fields from surface domains viewed by other techniques in only a planar $(x, y)$ projection have been studied in the $(x, z)$ plane perpendicular to the surface.
\end{abstract}

Keywords-Magnetic Domains; Grain-oriented Steel; Stray Field; Volumetric Scanning; Tunneling Magneto-Resistance; 3D structure

\section{INTRODUCTION}

Observation of magnetic domains based upon the Bitter technique [1] highlight the domain boundaries due to the presence of stray magnetic fields between domains. The Kerr effect [2], and observational techniques derived from both such as MOIF [3], reveal the body of domains through their interaction with polarized light. It might be expected that magneto-resistive sensors in a scanning microscope, measuring stray fields, would also highlight the edges of domains where the stray fields are presumed strongest. A three-dimensional scanning magnetic imaging system has been developed based on a Micromagnetics ${ }^{\circledR}$ STJ-020 MgO-based tunnelling magneto-resistance (TMR) sensor with a $2 \mu \mathrm{m} \times 4 \mu \mathrm{m}$ active area at a distance of $7 \mu \mathrm{m}$ from the tip edge (Fig. 1(a)).

The TMR sensor is mounted, alongside a Philtec ${ }^{\circledR} R C 20$ reflection-compensated fibre-optic displacement sensor for topology tracking, within a custom 3D-printed enclosure

Sponsored by Engineering and Physical Science Research Council, UK attached to a Parker Automation based 3-axis positioning arm with $\pm 1 \mu \mathrm{m}$ precision (Fig. 1). Similar systems have been used [4] to study geological samples [5] and custom permanent magnetic structures [6], but not for domain imaging at this resolution.

Examples of the three-dimensional study of magnetic fields have involved the use of 3-axis Hall-effect sensors [7] in a 3axis scanning system [6]. Such sensors must comprise three individual sensors mounted orthogonally, either physically or created on a single substrate [8]. Such complex sensors are limited by fabrication technologies to volumes in excess of 1 $\mathrm{mm}^{3}$, or are extremely expensive to create. A Hall-cross size of $700 \mathrm{~nm}$ is achieved [8] but with very limited scanning area.

Here, an alternative solution to investigating the structure of stray fields is proposed. The single-axis sensor (Fig. 1(b)) is mounted on a precision goniometer (Fig. 1(c)), by which the angle of the sensor can vary. The geometry of the sensor tip is illustrated in Fig. 2. If the variation in minimum sensor active area to surface distance $\left(\boldsymbol{z}_{o}+\delta \boldsymbol{z}\right)$ can be accounted for, and the coincidence of data and noise levels be confidently managed; then the components of the stray field magnetization vector $\boldsymbol{H}_{(x, z)}$ could be determined.

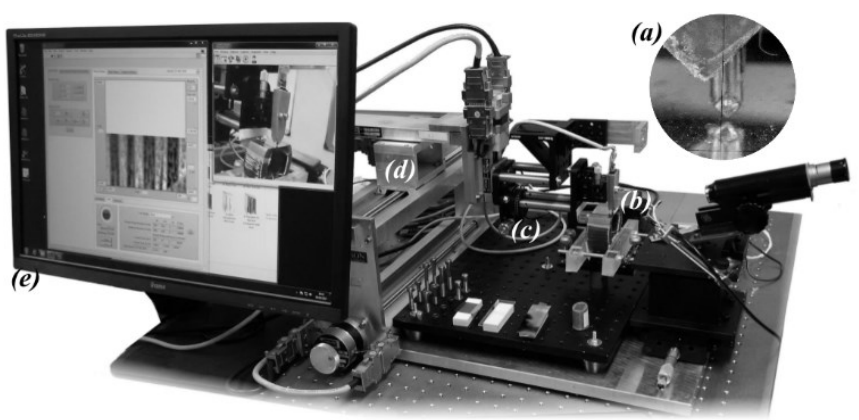

Fig. 1. The system developed is based on a MicroMagnetics ${ }^{\circledR}$ STJ-020 tunnelling magneto-resistance sensor with a Philtec RC20 fibre-optic displacement sensor for topology tracking. Figure insert (a) shows the sensor positioned above the reflective sample of electrical steel. The sensors (b) are mounted within a custom 3D-printed enclosure on a precision goniometer (c) attached to a Parker Automation based 3-axis positioning arm (d). A comprehensive interface (e) has been developed in NI LabVIEW ${ }^{\mathrm{TM}}$ to control the hardware and visualise and interrogate the resulting data. 


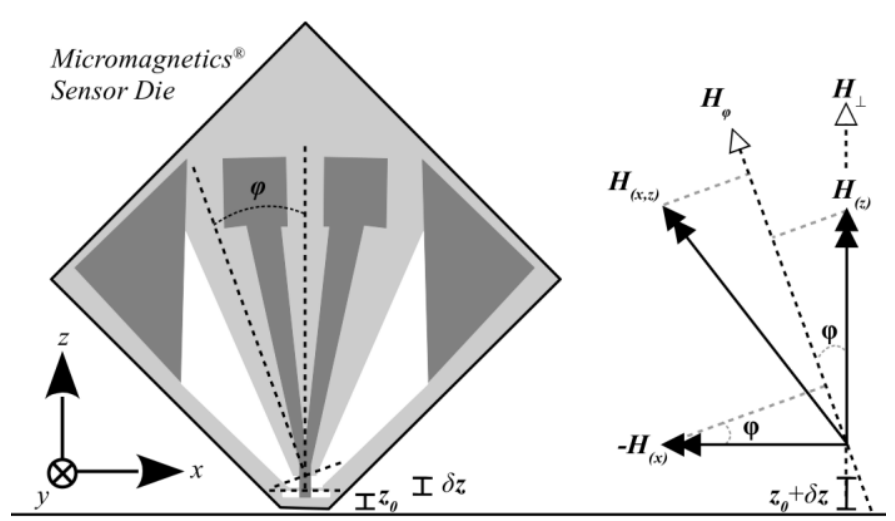

Fig. 2. Illustration of the geometry of the sensor tip. The tip of the sensor die has been polished to bring the active area within $7 \mu \mathrm{m}$ of the tip edge, resulting in a flat tip that enforces an increase in sensor to surface distance $\left(\boldsymbol{z}_{o}+\delta \boldsymbol{z}\right)$ as $\varphi$ increases. $\boldsymbol{H}_{(x)}$ and $\boldsymbol{H}_{(z)}$, the components of the field vector $\boldsymbol{H}_{(x, z)}$ can be derived from $\boldsymbol{H}_{\varphi}$, the field measured at angle $\varphi$ and $\boldsymbol{H}_{\perp}$, the field measured perpendicular once the difference in field due to $\delta \boldsymbol{z}$ is determined.

From two measurements of the perpendicular field $\boldsymbol{H}_{\perp}$ and the field $\boldsymbol{H}_{\varphi}$ at angle $\varphi$ then

$$
\boldsymbol{H}_{\varphi}=\boldsymbol{H}_{(z)} \cos (\varphi)-\boldsymbol{H}_{(x)} \sin (\varphi)
$$

where $\boldsymbol{H}_{(z)}$ and $\boldsymbol{H}_{(x)}$ are the components of $\boldsymbol{H}_{(x, z)}$, and thus

$$
\boldsymbol{H}_{(x)}=\left(\boldsymbol{H}_{(z)} \cos (\varphi)-\boldsymbol{H}_{\varphi}\right) / \sin (\varphi)
$$

and

$$
\boldsymbol{H}_{(z)}=\boldsymbol{H}_{\perp}-\boldsymbol{H}_{\delta z}
$$

with $\boldsymbol{H}_{\delta z}$ being the difference in stray field strength near the surface and at the height $z_{o}+\delta z$ above the surface.

\section{Planar (X,Y) SCAN OF GRAIN-ORIENTED (3\% SI) ELECTRICAL STEEL}

Planar $(\boldsymbol{x}, \boldsymbol{y})$ stray field scans of a polished uncoated sample of grain-oriented electrical steel $(3 \% \mathrm{Si} \mathrm{B} 32 \mathrm{~L})$ were made, with the sensor perpendicular to the sample surface (angle of sensor to vertical $\varphi=0^{\circ}$ ). Stray fields of between -116.0 and $272.0 \mathrm{~A} / \mathrm{m}$ were recorded normal to the sample surface, where a positive value indicates fields out from the surface, and negative into the surface. The resulting images are presented in Fig. 3 and Fig. 4. Grey (50\%) represents either true null-field or a field perpendicular to the sensor axis.

Each pixel in the resultant scans represents the mean of 20 samples of the field through the sensor's active area. Investigation was made into the best compromise between effective signal-to-noise reduction and increased scan time.

Main bar domains are clearly visible with evidence of internal Lancet [9] domains present. The domain images have greater qualitative similarity with Kerr effect (surface domain magnetization) observations than with Bitter technique (domain boundary) observations. Although scanned at a spatial resolution of $5 \mu \mathrm{m} / \mathrm{pixel}$, the system currently has limited resolving power for features smaller than $40-50 \mu \mathrm{m}$.
Three factors limit the system resolution; the scanner arm precision (calibrated to $\pm 1 \mu \mathrm{m}$ ), the width of the sensor's active area $(4 \mu \mathrm{m})$ and the sensor to sample-surface distance $\left(z_{o}\right)$. The sensor tip has been polished to bring the active area within $7 \mu \mathrm{m}$ of the tip edge. With the sensor vertical $\left(\varphi=0^{\circ}\right)$, $\boldsymbol{z}_{o}$ is still $9 \pm 1 \mu \mathrm{m}$ making this the dominant factor effecting the resolution. Continued efforts to control $\boldsymbol{z}_{0}+\delta \boldsymbol{z}$ further include better topology tracking using the Philtec ${ }^{\circledR} R C 20$ displacement sensor to directly monitor tip height.

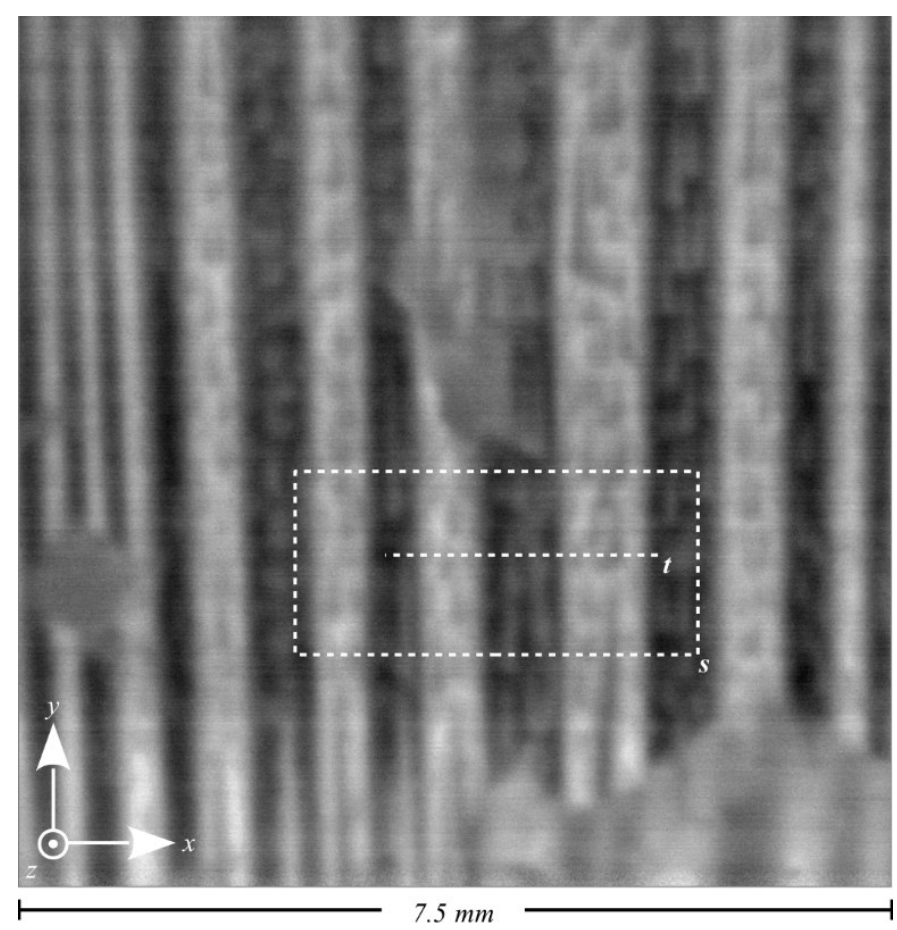

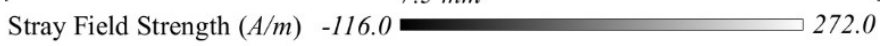

Fig. 3. Planar $(x, y)$ stray field scan of $7.5 \mathrm{~mm} \times 7.5 \mathrm{~mm}$ region of grainoriented electrical steel (3\% Si B32L) at a resolution of $10 \mu \mathrm{m} / \mathrm{pixel}$ with the sensor axis vertical $\left(\varphi=0^{\circ}\right)$. Stray fields of approximately $\pm 200 \mathrm{~A} / \mathrm{m}$ are recorded normal to the sample surface suggesting internal Lancet domains within the clear bar domains. Area "s" indicates the location of the sub-region (Fig. 4), and the line " $t$ " indicates the location of the (x,z) transects (Fig. 6).

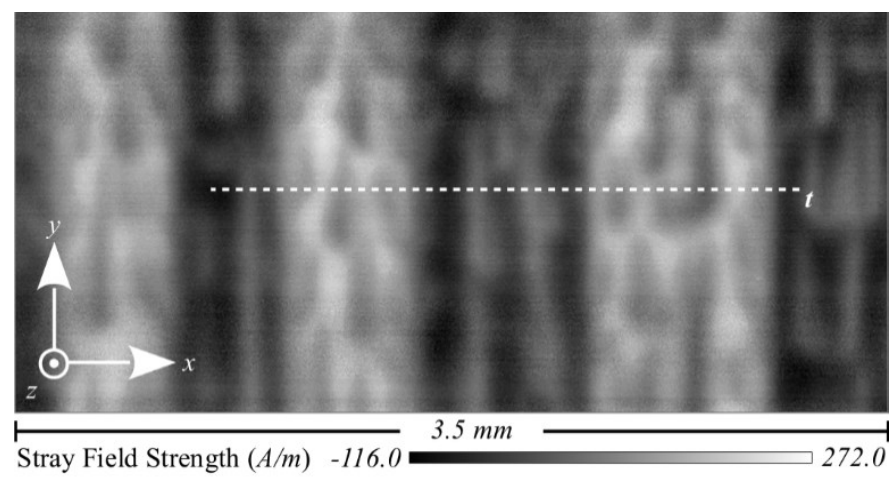

Fig. 4. Planar $(\boldsymbol{x}, \boldsymbol{y})$ stray field scan of the $3.5 \mathrm{~mm} \times 2.14 \mathrm{~mm}$ sub-region of grain-oriented electrical steel (3\% Si B32L) indicated in Fig. 3 at a resolution of $5 \mu \mathrm{m} / \mathrm{pixel}$. Stray fields of approximately $\pm 200 \mathrm{~A} / \mathrm{m}$ are recorded normal to the sample surface clearly indicating internal Lancet domains within the main bar domains. The location of the $(\boldsymbol{x}, \boldsymbol{z})$ transects (Fig. 6) is maked with line "t". 


\section{TRANSECT $(\mathrm{X}, \mathrm{Z})$ SCANS OF GRAIN-ORIENTED (3\% SI) ELECTRICAL STEEL}

Scans in the vertical $(\boldsymbol{x}, \boldsymbol{z})$ plane have been made along the $2.35 \mathrm{~mm}$ transect indicated in Fig. 3 and Fig. 4, to a height of $\boldsymbol{z}_{o}+0.1 \mathrm{~mm}$.

\section{A. Vertical $(\boldsymbol{x}, \boldsymbol{z})$ Transect Scan}

An illustration of the three-dimensional relationship between the vertical $(x, z)$ scan and the planar $(x, y)$ scan is presented in Fig. 5. The fields directly perpendicular to the sample surface are presented in the first $\left(\varphi=0^{\circ}\right)$ transect of Fig. 6. It can be seen that the fields emanating from the body of the smaller Lancet domains are limited in extent to approximately $40 \mu \mathrm{m}$ above the surface, before their effect is subsumed by the larger surrounding bar domains. The bar domains remain distinct to greater than $100 \mu \mathrm{m}$.

\section{B. Angled $(\boldsymbol{x}, \boldsymbol{z})$ Transect Scans}

Four further scans of the $2.35 \mathrm{~mm} \times 0.1 \mathrm{~mm}$ transect, with a sensor axis angle $\varphi$ of $5^{\circ}, 10^{\circ}, 15^{\circ}$ and $20^{\circ}$ to the vertical, are also presented in Fig. 6 . The intention is to determine the shape of the fields emanating from the Lancet domains and indicate how they may close back to the surface of the sample. At present, because it is difficult to distinguish between zero field or field perpendicular to the sensor axis the, results presented in Fig 6 have two possible causes; a rotation of the field vector compared with the sensor axis, or an $\mathrm{x}$-axis positional drift (less than $1 \mu \mathrm{m}$ ) coupled with a decrease in field magnitude caused by the enforced increase $\delta \boldsymbol{z}$ (Fig. 2) due to the increase in $\varphi$.

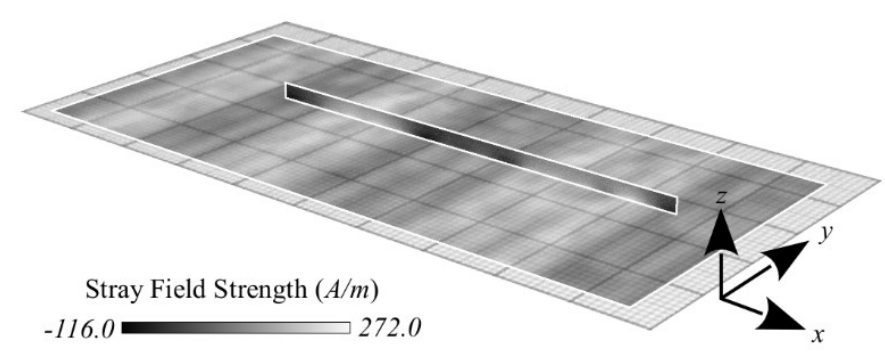

Fig. 5. Illustration of the three-dimensional relationship between the vertical $(\mathbf{x}, \mathbf{z})$ scans and the planar $(\mathbf{x}, \mathbf{y})$ scan. The $(\mathbf{x}, \mathbf{y})$ plane is presented at $50 \%$ opacity to emphasise the $(\mathbf{x}, \mathbf{z})$ plane.

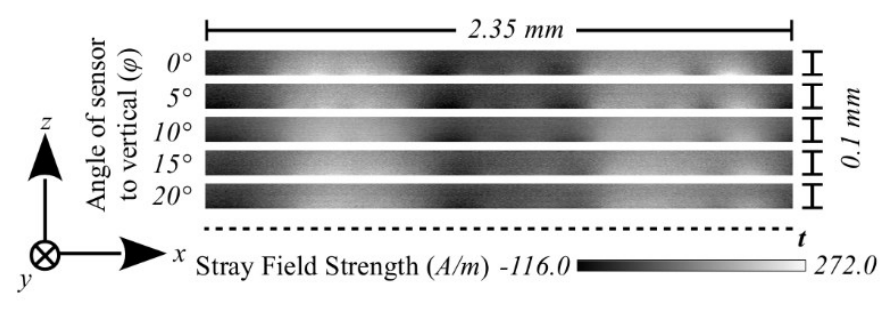

Fig. 6. Five $(\boldsymbol{x}, \boldsymbol{z})$ scans of the same $2.35 \mathrm{~mm} \times 0.1 \mathrm{~mm}$ transect, indicated by " $\mathrm{t}$ " in Fig. 4, with sensor axis angle $\varphi$ of between $0^{\circ}$ and $20^{\circ}$ to the vertical. The transects have an $\mathrm{x}$-axis resolution of $5 \mu \mathrm{m} / \mathrm{pixel}$ and $\mathrm{z}$-axis resolution of $2 \mu \mathrm{m} /$ pixel with $5: 2$ ratio non-square pixels to maintain the correct relative dimensions. The resulting drift of the field patterns have two possible causes, discussed in the text, which are currently difficult to isolate.

\section{CONCLUSIONS}

A sample of grain-oriented electrical steel $(3 \% \mathrm{Si})$ has been successfully scanned, indicating Lancet domain structures of width $100-150 \mu \mathrm{m}$ within bar domains of width $600-800$ $\mu \mathrm{m}$. The fields emanating from the body of these domains are shown to be $\pm 200 \mathrm{~A} / \mathrm{m}$ and extend $40-100 \mu \mathrm{m}$ above the surface of the sample.

With the minimum sensor active area to surface distance $\boldsymbol{z}_{o}$ of $9 \pm 1 \mu \mathrm{m}$, and the fields of some of the smaller Lancet domain fields shown to extend no more than $z_{o}+40 \mu \mathrm{m}$ from the surface of the sample (Fig. 6), this distance represents a failure to resolve the lowest $16 \%$ of the field. When $\varphi$ is increased, $\delta \boldsymbol{z}$ is enforced further by the flat edge of the tip.

Levels of noise remain in the data which, while qualitatively manageable by eye, cause difficulties for stricter quantitative geometric calculations. More work is needed to manage noise levels and the error margins in coincidence of repeated scans, before the components of the field at each point can be confidently isolated.

However, for the first time, the stray fields from surface domains viewed by other techniques $[1,2,3]$ in only a planar $(\boldsymbol{x}, \boldsymbol{y})$ projection have been studied in the $(\boldsymbol{x}, \boldsymbol{z})$ plane perpendicular to the surface. The strength and extent of the fields have been quantified and progress made towards demonstrating their shape, and how it varies spatially in the surface boundary layer with the underlying domain structure.

\section{ACKNOWLEDGMENT}

The authors wish to gratefully acknowledge the Engineering and Physical Science Research Council, UK for supporting this research through a studentship and Doctoral Training Grant (Ref EP/M507842/1).

\section{REFERENCES}

[1] F. Bitter, "Experiments on the nature of ferromagnetism", Physical Review, vol. 41, 1932, pp. 507-515

[2] J. Kerr, "On the rotation of the plane of polarization by reflection from the pole of a magnet", Phil. Mag. 5th Series, vol. 3, 1877, pp. 321-343

[3] H. Richert, H. Schmidt, S. Lindner, B. Wenzel, R. Holzhey and R. Shäfer, "Dynamic Magneto-Optical Imaging of Domains in GrainOriented Electrical Steel”, Steel Research, vol. 87, 2016, pp. 232-240

[4] G. Shaw, R.B.G. Kramer, N.M. Dempsey and K. Hasselbach, "A scanning Hall probe microscope for high resolution, large area, variable height magnetic field imaging", AIP Review of Scientific Instruments

[5] E.A. Lima, A.C. Bruno, H.R. Carvalho and B.P. Weiss, "Scanning magnetic tunnel junction microscope for high-resolution imaging of remanent magnetization fields", Meas. Sci. Technol., vol. 25, 2014, pp. $1-14$

[6] C. Huber, et al., "3D Print of Polymer Bonded Rare-Earth Magnets, and 3D Magnetic Field Scanning With an End-User 3D Printer", Applied Physical Letters, vol. 109, 2016, pp. 162401-1-162401-4

[7] J.B. Schell, et al., "CMOS 3D Hall probe for magnetic field measurement in MRI scanner", IEEE $10^{\text {th }}$ International New Circuits and Systems Conference, 2012, pp. 517-520

[8] M. Dede, R. Akram, A. Oral, "3D scanning Hall probe microscopy with $700 \mathrm{~nm}$ resolution”, Applied Physics Letters, vol. 109, 2016, pp. 182407-1-182407-5

[9] S. Shin, R. Schafer, B.C. de Cooman, "Grain boundary penetration by Lancet domains in Fe-3\%Si Grain-oriented steel", IEEE Transactions on Magnetics, vol. 46, issue 9, 2010, pp. 3574-3581 